\title{
Yannis KAKRIDIS
}

Universität Bern, Institut für slavische Sprachen und Literaturen (Schweiz, Bern)

kakridis@issl.unibe.ch

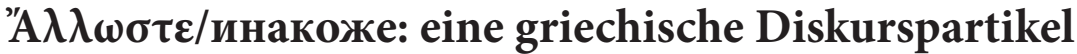 und ihr kirchenslavisches Äquivalent ${ }^{1}$}

Nicht selten macht man sich als Slavist daran, einen Text zu edieren, der aus dem Griechischen ins Kirchenslavische übersetzt wurde, und muss sich schliesslich mit der handschriftlichen Überlieferung seiner Vorlage beschäftigen, weil einen deren Edition im Stich lässt. Ähnlich — wenn auch weniger oft — kann es passieren, dass man sich an die Analyse eines kirchenslavischen Lexems macht und dabei feststellt, dass die lexikographische Beschreibung seiner griechischen Übersetzungsäquivalente unvollständig ist. Die vorliegende Studie ist einem solchen Fall gewidmet. Sie nahm ihren Ausgang in der Kartothek des Wörterbuchs der russischen Sprache des 11.-14. Jahrhunderts, die ich während meines Moskauaufenthaltes im Herbst 2018 benutzen durfte. Nicht nur für diese Möglichkeit, sondern auch für fachlichen Rat, moralische Unterstützung und anregende Gespräche über viele Jahre hinweg sei dem Jubilar herzlich gedankt!

Neugriechische Wörterbücher verzeichnen die ,adversative Konjunktion“ (in moderner Terminologie: die Diskurspartikel) $\alpha \dot{\lambda} \lambda \omega \sigma \tau \varepsilon$ mit einer Bedeutung, die dem französischen d'ailleurs, dem englischen besides oder dem leicht veralteten

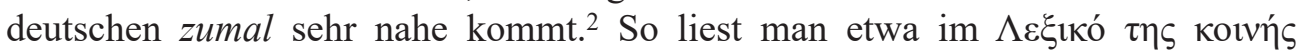

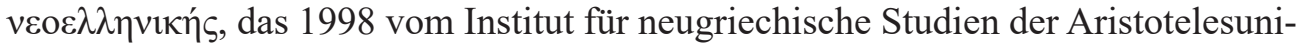
versität von Thessaloniki herausgegeben wurde, folgenden Eintrag:

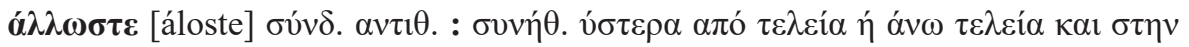

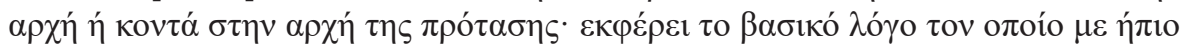

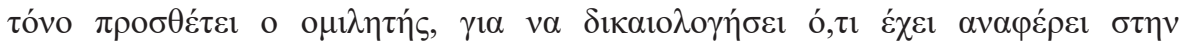

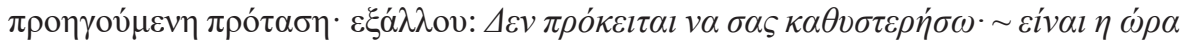

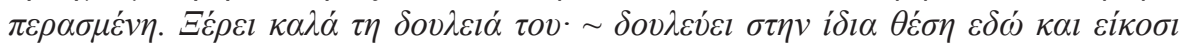

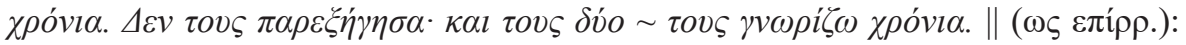

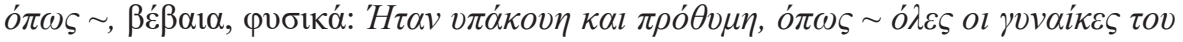

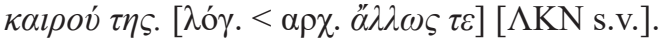

ÜBERSETZUNG: á $\lambda \lambda \omega \sigma \tau \varepsilon$ [áloste] adversative Konjunktion: gewöhnlich nach einem Punkt oder Semikolon und am Satzanfang oder nahe am Satzanfang; nennt den Hauptgrund, den der Sprecher in ruhigem Ton anführt, um das, was er im vorangehenden Satz gesagt hat, zu rechtfertigen; $\varepsilon \xi \dot{\alpha} \lambda \lambda$ ov 〈ausserdem〉: $\Delta \varepsilon v \pi \rho o ́ \kappa \varepsilon ı \tau \alpha l$ $v \alpha \sigma \alpha \varsigma \kappa \alpha \theta v \sigma \tau \varepsilon \rho \eta \dot{\sigma} \sigma \omega \cdot \sim$ siv $\alpha l \eta \dot{\eta} \rho \alpha \pi \varepsilon \rho \alpha \sigma \mu \varepsilon \dot{v} \eta$ 〈Ich werde Sie nicht aufhalten, zu-

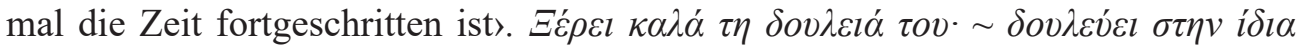

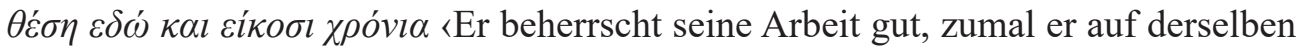

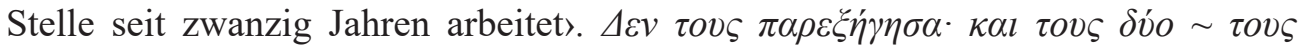

1 Dieser Aufsatz ist im Rahmen des Projektes „The diatribe in Medieval Orthodox Slavic literature“ entstanden, das vom Schweizerischen Nationalfonds finanziert wird (Nr. 182376). Für sorgfältige Durchsicht des Textes und Diskussion danke ich Herrn Dr. E. Bounatirou.

2 Vgl. die detaillierte Analyse von frz. d'ailleurs in [Ducrot et al. 1980: 193-232]. 


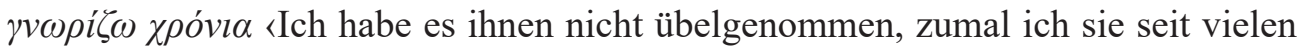

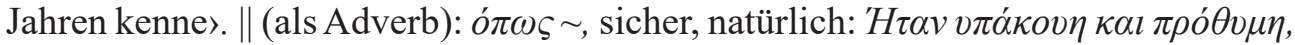

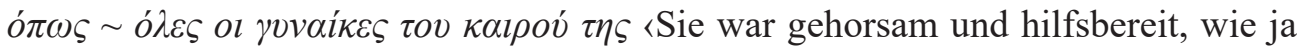
alle Frauen ihrer Zeit> [gelehrt $<$ altgriechisch $\ddot{\alpha} \lambda \lambda \omega \varsigma \tau \varepsilon$ ].

Im Wörterbuch von D. B. Dimitrakos wird von $\alpha \ddot{\lambda} \lambda \omega \omega \sigma \tau \varepsilon$ auf das Lemma von $\alpha \ddot{\lambda} \lambda \omega \varsigma$ verwiesen, wo folgendes steht:

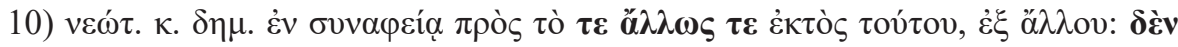

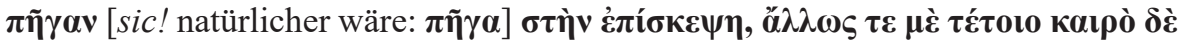

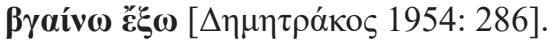

ÜBERSETZUNG: 10) neuzeitlich, demotisch; in Verbindung mit $\tau \varepsilon: \ddot{\alpha} \lambda \lambda \omega \varsigma \tau \varepsilon$ ausserdem, übrigens: Sie gingen [wohl Druckfehler für: Ich ging] nicht zu Besuch, ich gehe bei solchem Wetter ohnehin nicht aus.

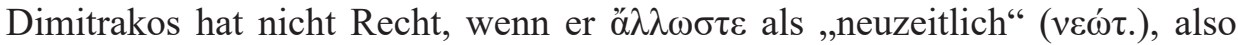
nachmittelalterlich, und „demotisch“ $(\delta \eta \mu$.) bezeichnet. Näher an die Wahrheit kommen die Autoren des Wörterbuchs des Instituts für neugriechische Studien, die dem Wort eine gelehrte Herkunft attestieren ( $\lambda \circ \gamma$.) und auf altgriechisch $\alpha \ddot{\lambda} \lambda \omega \varsigma \tau \varepsilon$

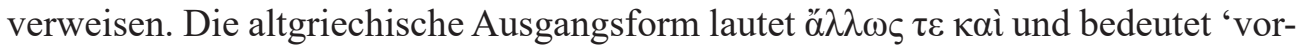
züglich, zumal', oder wörtlich: 'sowohl auf andere Weise / aus anderen Gründen, als auch weil...' Sie liegt etwa an folgender Stelle im platonischen «Phaedros» vor (247cd):

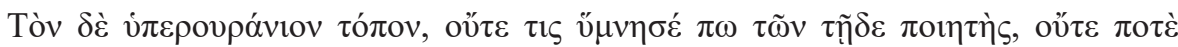

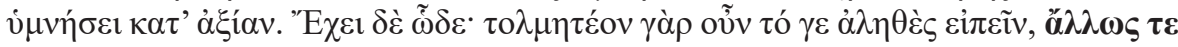

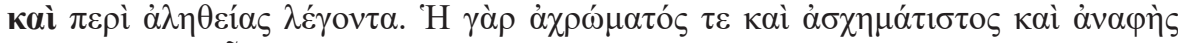

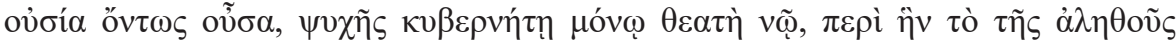

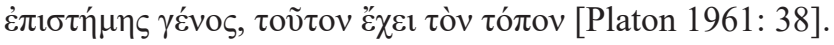

ÜBERSETZUNG: Den überhimmlischen Ort aber hat noch kein irdischer Dichter je gebührend besungen, und das wird auch niemals einer tun. Es verhält sich aber damit so - denn man sollte es wagen, die Wahrheit zu sagen, zumal dann, wenn man über die wahre Natur der Dinge spricht — : Das Sein, das ohne Farbe und Gestalt und nicht zu berühren, sichtbar nur der Vernunft als dem Lenker der Seele, allein Gegenstand wahrer Erkenntnis, das ist an diesem Ort [Platon 1997: 32].

Das kaì kann von $\alpha \ddot{\lambda} \lambda \omega \varsigma \tau \varepsilon$ durch andere Wörter getrennt werden, wie an folgender Stelle aus den Memorabilien des Xenophon $(1,2,59)$ :

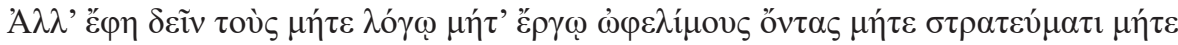

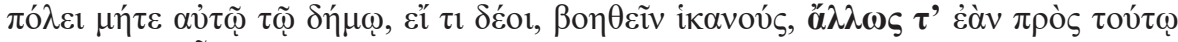

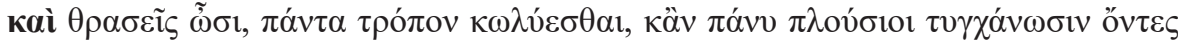
[Xenophon 1987: 42].

ÜBERSETZUNG: Sondern er [Sokrates] sagte, wer weder durch Rat noch durch Tat nützlich sei und weder dem Heer noch dem Staat noch dem Volk selbst helfen könne, falls es nottue, und sofern er sonst dazu auch noch frechen Sinnes sei, der müsse auf jede Weise in Schranken gehalten werden, wenn er auch zufällig sehr reich sei [Xenophon 1987: 43]. 
Schliesslich kann das кaì ganz ausgelassen werden. Einer der ältesten Belege dafür findet sich im Ödipus Rex (1114):

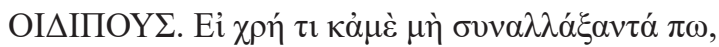

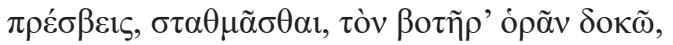

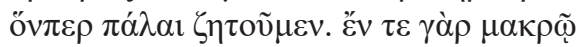

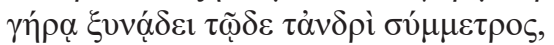

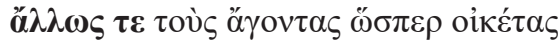

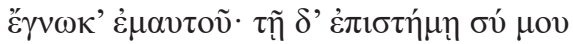

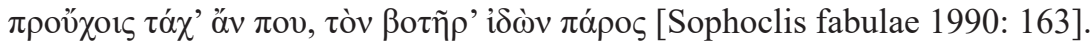

ÜBERSETZUNG: Wofern auch ich, der nie mit ihm zu tun gehabt, Ihr Ältesten, vermuten darf, mein ich, den Hirten $\mathrm{Zu}$ sehn, nach dem wir lange suchen. Denn im hohen Alter stimmt er mit diesem Manne überein, Und überdies erkenn ich, die ihn führen, als meine Diener. Doch bist du vielleicht An sicher Kenntnis mir voraus, da du Den Hirten früher schon gesehn [Sophokles 1968: 210].

Dieser in altgriechischer Zeit noch seltene Gebrauch von ö $\lambda \lambda \omega \varsigma \tau \varepsilon$ ohne $\kappa \alpha i ̀$ verbreitet sich allmählich und führt zur Entstehung der Diskurspartikel ố $\lambda \lambda \omega \sigma \tau \varepsilon$.

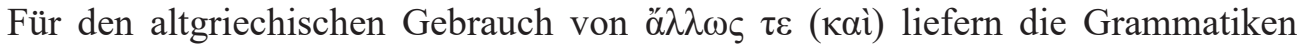
[Kühner 1904: 250] und die Wörterbücher [Pape 1954: 107] solide Informationen. Eine Reihe spätantiker Belege bietet der Thesaurus graecae linguae [Stephanus 1831: 1555]. Für die byzantinische Zeit stehen entsprechende Belegsammlungen oder Beschreibungen noch aus, wenn man von den kurzen Einträgen in den Wörterbüchern absieht, die die Byzantiner selbst verwendeten [Suidae lexicon 1928: 126].

Die Slavistik wird damit in eine gewisse Verlegenheit gebracht; denn Texte, die $\alpha \dddot{\alpha} \lambda \lambda \omega \varsigma \tau \varepsilon(\not \alpha \lambda \lambda \omega \sigma \tau \varepsilon)$ enthalten, wurden auch ins Kirchenslavische übersetzt und zwangen ihre Übersetzer, schon früh nach Äquivalenten Ausschau zu halten. Ein charakteristisches Beispiel findet man etwa in der Chronik des Georgios Hamartolos, die nach 963, aber vor der Mitte des 11. Jh. übersetzt wurde und sowohl süd- als auch ostslavische Züge aufweist [Pičchadze 2011: 26-28, 57-69]. Der fragliche Abschnitt ist der Polemik gegen den altgriechischen Polytheismus gewidmet. Die Frage, ob Zeus die Ursache des Regens ist, wird in diatribischer Manier als Dialog

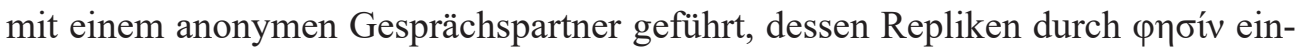
geleitet werden:

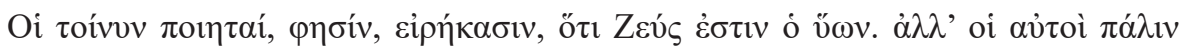

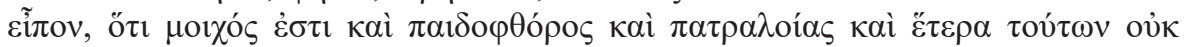

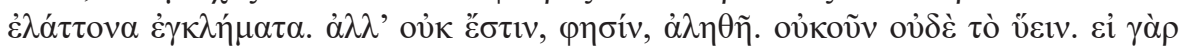

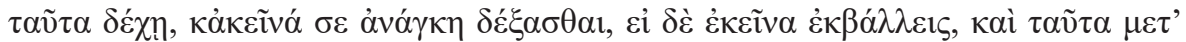

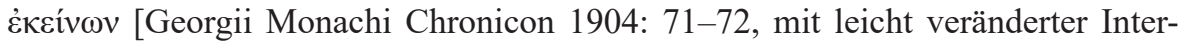
punktion].

ÜBERSETZUNG: Die Dichter, sagt er, haben gesagt, dass es Zeus ist, der regnen lässt. Aber dieselben Dichter sagten auch, dass er ein Ehebrecher und Knabenschänder und Vatermörder ist, und ähnliche, nicht geringere Verbrechen begangen hat. Aber, sagt er, das ist nicht wahr. Also auch nicht, dass er regnen lässt. Wenn du nämlich das akzeptierst, musst du notgedrungen auch das andere akzeptieren, wenn du aber jenes verwirfst, so mit ihm auch dies. 
Georgios Monachos lässt seinen Gegner behaupten, dass Zeus mit dem Äther gleichzusetzen sei, und widerlegt dies mit dem Hinweis, dass er dann ja vernunftlos sein müsste. Nach einer Reihe weiterer Argumente heisst es schliesslich:

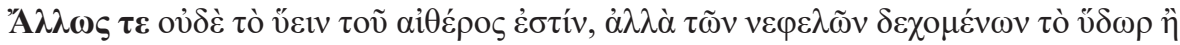

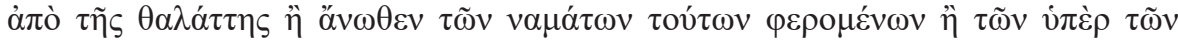

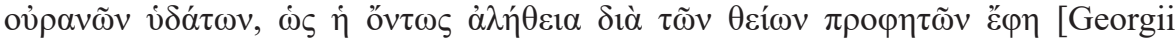
Monachi Chronikon 1904: 72].

ÜBERSETZUNG: Ausserdem ist es ja nicht der Äther, der regnet, sondern die Wolken, die das Wasser entweder vom Meer empfangen oder von oben, indem [dort] diese Ströme [d.h. wohl die Regenströme] fliessen oder das Wasser, das sich über dem Himmel befindet, wie die Wahrheit selbst durch die Propheten gesagt hat [cf. Ps. 148:4].

Der zitierte Abschnitt zeigt sehr schön die argumentative Funktion von $\ddot{\alpha} \lambda \lambda \omega \varsigma$ $\tau \varepsilon$. Es dient dazu, ein zusätzliches - manchmal auch zweitrangiges — Argument einzuleiten. In diesem Fall handelt es sich um ein Argument gegen die These, dass Zeus der Urheber des Regens sei. ${ }^{3}$

Der slavische Übersetzer hat $\alpha \lambda \lambda \omega \varsigma \tau \varepsilon$ Glied für Glied wiedergegeben:

Инако же ни дъждьвати юсть вьздоухоу, но облакомъ приюмлющимъ водоу ли от(ъ) мор^, ли горђ водамъ тъмъ възносимомъ ли выспрь н(е)б(ес)ныхъ водъ, ғако истиньнаға правда б(ож)ествьными пр(о)р(о)кы р(еч)е. [Istrin 1920: 68, in vereinfachter Orthographie]

Nach Ausweis des TLG [Thesaurus Linguae Graecae] kommt ö $\lambda \lambda \omega \varsigma \tau \varepsilon$ in der Chronik des Hamartolos noch zweimal vor, beide Male allerdings in erweiterter

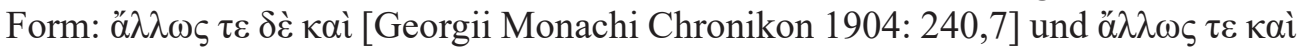
$[254,15]$. An beiden Stellen wird es mit инако же (и) übersetzt [Istrin 1920: 170,8 und 179,3$]$.

Dieselbe Lösung hat der Übersetzer von Epiktets Encheiridion gewählt. Das Encheiridion - eine Zusammenstellung der wichtigsten Lehren von Epiktet wurde in der sog. Paraphrasis christiana im 10. Jh. aus dem Griechischen ins Altbulgarische (Altkirchenslavische) übersetzt [Bulanin 1991: 96-37; Bulanin 2014: 268, 435-448]. Es enthält vor allem Anweisungen, wie der stoische Philosoph (in der Paraphrasis: der Mönch) auf die Widernisse des Lebens reagieren sollte. So heisst es im 56. Kapitel, man müsse, wenn jemand Böses tut oder übel redet, sich in Erinnerung rufen, dass er das nur tut, weil er es für sich selbst von Vorteil hält. Man kann jedoch niemanden dazu bringen, den eigenen Vorteil für den eines anderen aufzugeben, und man sollte es deshalb auch gar nicht versuchen. Ein solcher Versuch würde ja gegen die Hauptregel der stoischen Philosophie verstossen - die

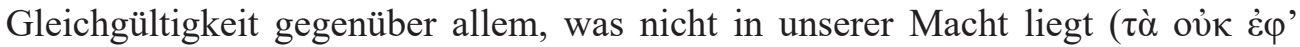
$\dot{\eta} \mu \mathrm{i} v)$. Nach diesem zentralen Argument wird ein zweites nachgeschoben:

${ }^{3}$ Die Übersetzung durch „,dalee“ in [Matveenko, Ščegoleva 2011, 1:115] ist angemessen, allerdings zeigt der Kommentar im Begleitband [Matveenko, Ščegoleva 2011, 2: 180], dass die Übersetzer damit nur $\alpha \lambda \lambda \lambda \omega \varsigma$ widergeben wollten. Die Variante никако ist sicher sekundär. Wahrscheinlich war der Sinn von инако же an dieser Stelle schon in altrussischer Zeit den allerwenigsten Lesern klar. 


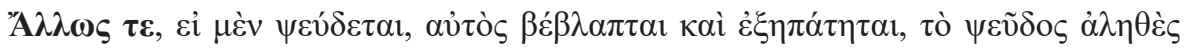
vouíбas. [Boter 1999: 384]

ÜBERSETZUNG: Des weiteren: wenn er lügt, so hat er selbst den Schaden und ist betrogen, da er die Lüge für wahr gehalten hat.

In der Edition von D. Bulanin lautet der slavische Text folgendermassen:

Инако же, тебе облыгаеть - себе же пакостьствоуеть и прельщает сА, лъжю истиноу имњвъ. [Bulanin 1991: 317, in vereinfachter Orthographie und mit Veränderung der Interpunktion]

Nicht alle Übersetzer geben $\alpha \varkappa \lambda \lambda \omega \varsigma \tau \varepsilon$ mit инако же wider. Eine Reihe anderer Äquivalente finden wir in der Übersetzung der «Christlichen Topographie» des Kosmas Indikopleustes, über deren Entstehungszeit noch keine Klarheit herrscht [Pičchadze 2011: 30-31]. Auch wenn die «Topographie» in der zeitgenössischen Forschung als «Beschreibung des Erdkreises, von Ländern, Völkern, der Natur und des Tierreichs» [ibid.] gilt, handelt es sich doch um einen Text mit polemischer Ausrichtung und vielen argumentativen Passagen. In drei solchen Passagen finden wir ő $\lambda \lambda \omega \varsigma \tau \varepsilon$. Wir wollen hier nur die erste von ihnen anführen. Kosmas rechtfertigt sich gegenüber dem Adressaten seines Werkes, einem gewissen Pamphilos, dafür, dass sich die Abfassung der «Topographie» verzögert habe:

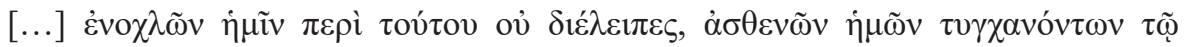

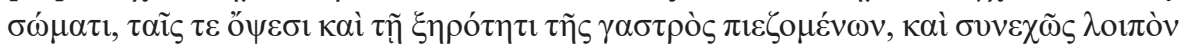

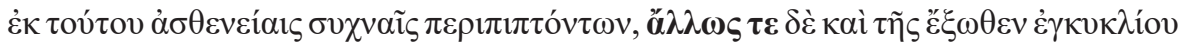

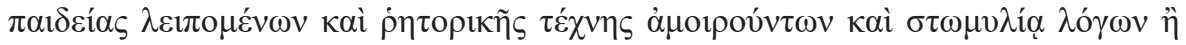

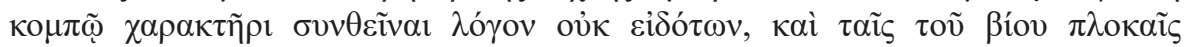

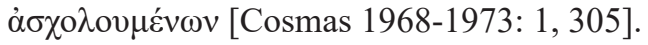

ÜBERSETZUnG: Du [Pamphilos] hast nicht davon abgelassen, mich [wörtlich: uns] daran [d.h. an die Abfassung meines Werkes] zu erinnern, obwohl mein Körper geschwächt war, ich an Sehstörungen und Obstipation litt und deshalb immer wieder krank wurde; überdies fehlt mir die weltliche Allgemeinbildung und die Kunst des Rhetors; ich bin nicht in der Lage, einen Text mit fliessender Rede und prächtigem Putz zu verfassen, und werde von Sorgen um meinen Lebensunterhalt abgelenkt.

In der kirchenslavischen Übersetzung lautet dieser Abschnitt folgendermassen:

[...] и понужаа насъ о семь не от(ъ)ступиль еси, намь немощномь сущемь тъломь, видом же и соухотою оутробы обдержимомь, и часто к томоу от(ъ) болъзнеи частыхь падающю, еще же и внъшнла хытрости наказаниа не имыи, ни риторьска хоудожества, ни красотою слова сьставити въдыи, и житиискых(ъ) соплетении оупражненоу [...] [Kniga naricaema Koz'ma Indikoplov 1997: 58, in vereinfachter Orthographie].

Еще же ist ein verständlicheres und deshalb — zumindest nach den modernen Massstäben der Übersetzungskritik — gelungeneres Äquivalent von ő $\lambda \lambda \omega \varsigma \tau \varepsilon$ als die Lehnübersetzung инако же. Leider ist der Übersetzer nicht dabei geblieben, sondern hat an den zwei anderen Stellen, wo im Text der "Topographie» ö $\lambda \lambda \omega \varsigma \tau \varepsilon$ 
vorkommt, zu anderen Lösungen seine Zuflucht genommen. Seine Übersetzungen

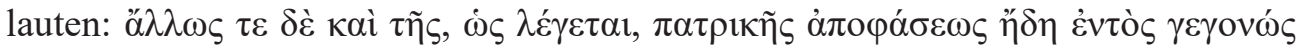
[Wolska 1968-1973: 2, 121] = Но пакы нако ж(е) кто г(лаго)л(е)ть от(ь)чю от(ь)

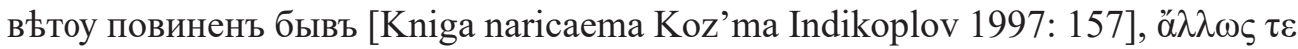
$\delta \grave{\varepsilon}$ [Wolska 1968-1973: 3, 121] = но нако же и [Kniga naricaema Koz'ma Indiko-

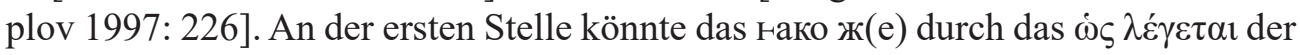
Vorlage erklärt werden, an der zweiten wurde offensichtlich $\alpha \prime \lambda \lambda \omega \varsigma \tau \varepsilon \delta \dot{\varepsilon}$ als $\dot{\alpha} \lambda \lambda \lambda^{\prime} \dot{\omega} \varsigma$ $\tau \varepsilon \delta \grave{\varepsilon}$ missverstanden und Wort für Wort (mit Vertauschung von $\tau \varepsilon$ und $\delta \grave{\varepsilon}$ ) übersetzt — soweit man natürlich überhaupt auf der Grundlage einer einzigen Abschrift ein Urteil über Fragen der Übersetzungstechnik abgeben kann.

Im 14. scheint sich инакоже als festes Äquivalent für $\alpha \varkappa \lambda \lambda \omega \varsigma \tau \varepsilon(\alpha ̋ \lambda \lambda \omega \sigma \tau \varepsilon)$ etabliert zu haben. Wir finden es mehrmals in der Übersetzung des Corpus Areopagiticum, die der Mönch Isaija 1371 fertiggestellt hat. So versucht etwa (Pseudo-)Dionysios Areopagites im 4. Kapitel seines Traktats «De divinis nominibus» den Beweis zu erbringen, dass sogar die Dämonen nicht von Natur aus böse sind, und führt zu diesem Zweck eine ganze Reihe von Argumenten an. Der Übergang vom einen Argument zum anderen findet mit Hilfe von Diskurspartikeln statt, unter denen wir auch $\alpha \varkappa \lambda \lambda \omega \varsigma \tau \varepsilon$ finden:

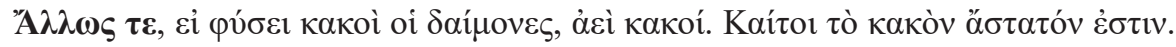

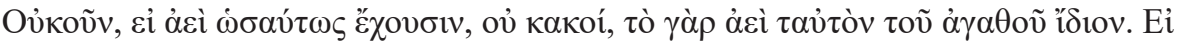

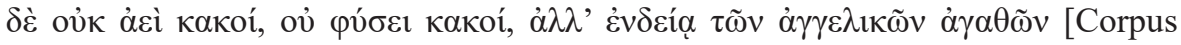
Dionysiacum 1990: 171].

ÜBERSETZUNG: Ferner: Wenn die Dämonen von Natur aus schlecht sind, dann sind sie schlechthin immer böse. Nun ist aber das Böse unstetig. Folglich sind die Dämonen, wenn sie sich immer auf die gleiche Weise verhalten, nicht böse, da es nur dem Guten eigen ist, immer dasselbe zu sein. Wenn sie aber nicht immer böse sind, dann sind sie nicht von Natur böse, sondern durch einen Mangel des den Engeln eignenden Guten. [Pseudo-Dionysius Areopagita 1988: 61; das «Ferner»am Anfang wurde von uns hinzugefügt]

In der serbisch-kirchenslavischen Übersetzung des Mönches Isaija sieht diese Stelle folgendermassen aus:

Инако же, аще юс(ть)ствомь злы бъсове, присно злы. а паче зло, нестоғателно юсть. проче оубо аще присно такожде имоуть, не злы. юже бо присно такожде бл(а)гомоу своиствно. аще ли не присно злы, не юс(ть)ствомь злы, нь оскоудъниюмь агг(е)льскыих(ь) бл(а)гьь [Goltz, Prochorov 2010-2013: 2, 308].

Инакоже tritt als Äquivalent von $\alpha ̋ \lambda \lambda \omega \varsigma \tau \varepsilon$ im Corpus Dionysiacum mehrmals auf. Hier sind die entsprechenden Stellen nach der ältesten Abschrift (Hilferding 47): a) im Text: 6b,2; 143a,4; 146a,12; 209b,1; 321b,11 und b) in den Scholien: 36a, 12; 41a,39; 59b,43; 74b,22.

Eine Reihe weiterer interessanter Beispiele finden wir in den Synaxarien zum Triodion und Pentekostarion des Nikephoros Kallistos Xanthopoulos, die um die Mitte des 14. Jh. vom bulgarischen Übersetzer Zakchäus dem Philosophen ins Kirchenslavische übertragen wurden. In seinen Synaxarien geht Nikephoros oft auf die 
Gründe ein, die die Väter zur Wahl einer bestimmten Perikope bewogen haben. Für

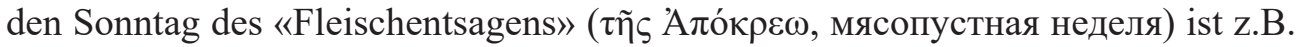
die Lesung aus Matthäus 16, 9-20 vorgesehen, die das Jünste Gericht behandelt. Nikephoros erklärt, das geschehe, damit die Seelen nicht durch die Gleichnisse vom Zöllner und dem Pharisäer bzw. dem verlorenen Sohn (die an den beiden vorangehenden Sonntagen gelesen wurden) dazu verführt werden, sich in der Hoffnung auf die Barmherzigkeit Gottes der Sünde hinzugeben, sondern damit sie sich vergegenwärtigen, dass er ein gerechter Richter ist, der jedem Menschen nach seinen Taten vergelten wird. Danach fügt er noch einen Grund an. Der Samstag vor dem Sonntag des Fleischentsagens ist ein Tag allgemeinen Totengedenkens. Es ziemt sich deshalb, nach den Seelen der Verstorbenen auch ihres Richters zu gedenken:

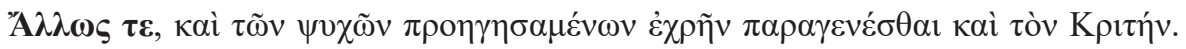

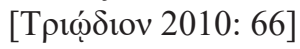

ÜBERSETZUNG: Ausserdem musste, nachdem die Seelen vorangegangen waren, auch der[en] Richter erscheinen.

Im mittelbulgarischen Triodion (Cod. Sin. Slav. 23, л. 50a) lautet diese Stelle folgendermassen:

Инако же, и д(оу)шамъ пръдъварившемъ, достоаше приит-и соудии. [Тасева 2010: 201]

Die übrigen Stellen, an denen инакоже als Äquivalent von $\alpha \dot{\lambda} \lambda \omega \sigma \tau \varepsilon$ in der mittelbulgarischen Übersetzung der Synaxarien verwendet wurde, sind: Sin. 23, 38а [Тасева 2010: 181], Sin. 23, 39v [187], Sin. 23, 88v [223], Sin. 24, 250a [459], Sin. 24, 371a [515].

Unsere bisherigen Beispiele stammten alle von übersetzten Texten. Es scheint aber, dass es zumindest einem slavischen Autor des Mittelalters gelungen ist, sich ein Bild von der Bedeutung von инакоже zu machen und diese Diskurspartikel in einem eigenen Text zu verwenden. Dieser Autor ist Filofej von Pskov, und sein Text das berühmte Sendschreiben and Misjur" Munechin «Gegen die Astrologen und die Lateiner» aus dem Jahr 1523 oder 1524, das als ältester Beleg für die Doktrin von «Moskau dem dritten Rom» gilt. Filofej versucht in seinem Sendschreiben unter anderem, das Argument zu widerlegen, dass die «Lateiner» (d.h. die Westeuropäer) gar nicht Häretiker sein können, da ja sonst Gott ihr Reich (entweder das Heilige Römische Reich oder überhaupt die Staaten Westeuropas) der Zerstörung anheimgegegeben hätte. Dazu führt er eine Reihe von Gegenargumenten an. Das letzte von ihnen lautet:

Инако же Ромеиское царьство неразрушимо, яко Господь в Римскую власть написася. [Sinicyna 1998: 343].

ÜBERSETZUnG: Ausserdem ist das Römische Reich unzerstörbar, weil sich der Herr unter der Herrschaft Roms registrieren liess.

Eine vollständige Rekonstruktion von Filofejs Argumenten braucht an dieser Stelle nicht geleistet zu werden. Es sei lediglich angemerkt, dass dieses letzte Argu- 
ment die Rolle einer «condition of exception of rebuttal» [Toulmin 2003: 93-94] spielt. Der Wohlstand eines Staates zählt als Argument für die Rechtgläubigkeit seiner Untertanen — aber nur, wenn dieser Staat prinzipiell zerstörbar ist. Das Römische Reich ist dies jedoch wegen seiner providentiellen Verbindung mit der Inkarnation Christi nicht.

Das Material, das hier vorgestellt wurde, legt nahe, in den Wörterbüchern des Kirchenslavischen bzw. Altrussischen auch инакоже als eigenes Lemma aufzunehmen. ${ }^{4}$ Dazu wären allerdings auch die Stellen zu analysieren, an denen инако же als Äquivalent anderer griechischer Ausdrücke auftritt — etwa von $\alpha \ddot{\lambda} \lambda \lambda \omega \varsigma \delta \grave{\varepsilon}$, das offensichtlich ebenfalls in manchen Kontexten eine ähnliche Funktion wie ö $\lambda \lambda \omega \varsigma \tau \varepsilon$ erfüllen konnte. Aber das wäre schon ein Thema für eine andere Untersuchung.

\section{QUELLEN}

Boter G. The Encheiridion of Epictetus and its three Christian Adaptations. Transmission and Critical Editions. Leiden, Boston, Köln: Brill, 1999. 446 S.

Corpus Dionysiacum I. Pseudo-Dionysius Areopagita. De divinis nominibus. Hsg. von Beate Regina Suchla. Berlin, New York: Walter de Gruyter, 1990. 238 S.

Cosmas Indicopleustès. Topographie chrétienne. Introduction, texte critique, illustration, traduction et notes par W. Wolska-Conus. T. 1-3. Paris: Les Éditions du Cerf, 1968-1973.

Georgii Monachi Chronicon edidit Carolus de Boor. Vol. I. Lipsiae: In aedibus B. G. Teubneri, 1904. $804 \mathrm{~S}$.

Goltz H., Prochorov G. M. Das Corpus des Dionysios Areiopagites in der slavischen Übersetzung von Starec Isaija (14. Jahrhundert). Bd. 1-5. Freiburg i. Br.: Weiher, 2010-2013.

Istrin V. M. Chronika Georgia Amartola v drevnem slavjanorusskom perevode. Tekst, issledovanie i slovar'. T. 1. Tekst. Petrograd: Rossijskaja gosudarstvennaja akademičeskaja tipografija, 1920. $612 \mathrm{~S}$.

Kniga naricaema Koz'ma Indikoplov. Izdanie podgotovili V. S. Golyšenko, V. F. Dubrovina. Moskva: Indrik, 1997. $774 \mathrm{~S}$.

Matveenko V., Ščegoleva L. Knigi vremennye i obraznye Georgija Monacha. V dvuch tomach. Moskva: Nauka, 2011. 480 S.

Platon. Euvres complètes. T. IV - 3e partie. Phèdre. Texte établi et traduit par Léon Robin. Paris: Les belles lettres, 1961. 196 S.

Platon. Phaidros. Übersetzung und Kommentar von Ernst Heitsch. 2., erweiterte Auflage. Göttingen: Vandenhoeck \& Ruprecht, 1997. 281 S.

Pseudo-Dionysius Areopagita. Die Namen Gottes. Eingeleitet, übersetzt und mit Anmerkungen versehen von Beate Regina Suchla. Stuttgart: Anton Hiersemann, 1988. 145 S.

Sophokles. Tragödien. Herausgegeben und mit einem Nachwort versehen von Wolfgang Schadewaldt. Zürich und Stuttgart: Artemis Verlag, 1968. 460 S.

Sophoclis fabulae. Recognoverunt brevique adnotatione critica instruxerunt H. Lloyd-Jones [...] et N. G. Wilson [...]. Oxonii: E Typographeo Clarendoniano, 1990. 431 S.

Suidae lexicon. Edidit Ada Adler. Pars I. Lipsiae: In aedibus B. G. Teubneri, 1928. 549 S.

Taseva $L$. Triodnite sinaksari v srednovekovnata slavjanska knižnina. Tekstologično izsledvane. Izdanie na Zakcheevija prevod. Slovoukazateli. Freiburg i. Br.: Weiher, 2010. 823 S.

TLG - Thesaurus Linguae Graecae [https://stephanus.tlg.uci.edu/index.php] (Aufgerufen am 26.02. 2020]

Xenophon. Erinnerungen an Sokrates. Griechisch-deutsch. Herausgegeben von Peter Jaerisch. München, Zürich: Artemis Verlag, 1977. 392 S.

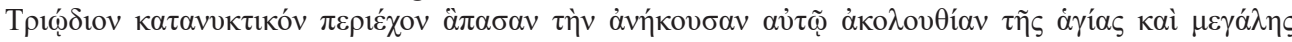

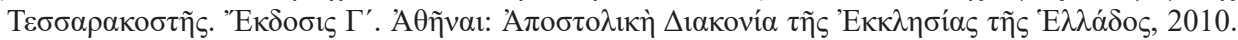
$1093 \mathrm{~S}$.

${ }^{4}$ Das einzige Wörterbuch, dass инакоже (wenn auch nur als Unterlemma von инако) enthält, ist [Miklosich 1862-65: 256]. 


\section{WÖRTERBÜCHER}

Miklosich Fr. Lexicon Palaeoslovenico-Graeco-Latinum. Vindobonae: Guilelmus Braumueller, 18621865. $1171 \mathrm{~S}$.

Pape W. Griechisch-deutsches Handwörterbuch. Nachdruck der dritten Auflage. Bearbeitet von M. Sengebuch. Bd. 1. A - K. Graz: Akademische Verlagsanstalt, 1954. 1548 S.

Stephanus H. Thesaurus graecae linguae, ab Henrico Stephano constructus. Post editionem anglicam [...] tertio ediderung Carolus Benedictus Hase et al. Volumen primum. Parisiis: Excudebat Ambrosius Firmin Didot, 1831. 2232 Sp.

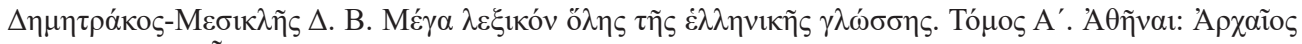

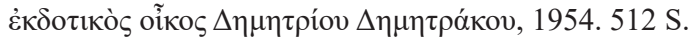

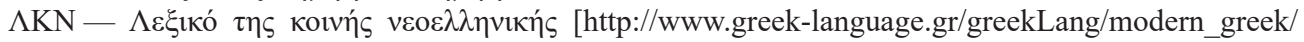
tools/lexica/triantafyllides/index.html] (aufgerufen am 25.02.2020)

\section{LITERATUR}

Bulanin D. M. Antičnye tradicii v drevnerusskoj literature XI-XVI vv. München: Verlag Otto Sagner, 1991. 465 S. (Slavistische Beiträge. Bd. 278).

Bulanin D. M. Katalog pamjatnikov drevnerusskoj pis'mennosti XI-XIV vv. (rukopisnye knigi). SPb.: Dmitrij Bulanin, 2014. $942 \mathrm{~S}$.

Ducrot O. et al. Les mots du discours. Paris: Les Éditions de Minuit, 1980. 240 S.

Kühner R. Ausführliche Grammatik der griechischen Sprache. Zweiter Teil: Satzlehre. Dritte Auflage in zwei Bänden. In neuer Bearbeitung besorgt von B. Gerth. Zweiter Band. Hannover, Leipzig: Hahnsche Buchhandlung, 1904. $714 \mathrm{~S}$.

Pičchadze A. A. Perevodčeskaja dejatel'nost' v domongol'skoj Rusi. Lingvističeskij aspekt / RAN, Institut russkogo jazyka im. V. V. Vinogradova. Moskva: Rukopisnye pamyatniki Drevnei Rusi, 2011. $403 \mathrm{~S}$.

Sinicyna N. V. Tretij Rim. Istoki i ėvoljucija russkoj srednevekovoj koncepcii (XV-XVII vv.). M.: Indrik, 1998. $410 \mathrm{~S}$.

Toulmin St. E. The Uses of Argument. Updated edition. (1 ${ }^{\text {st }}$ edition 1958). Cambridge: Cambridge UP, 2003. $247 \mathrm{~S}$. 